\title{
The impact of CoRoT on close binary research
}

\author{
Carla Maceroni (maceroni@oa-roma.inaf.it) \\ INAF - Osservatorio Astronomico di Roma, Monteporzio Catone, Italy \\ Ignasi Ribas (iribas@ieec.uab.es) \\ CSIC - Institut d'Estudis Espacials de Catalunya, Barcelona, Spain
}

September 28, 2005

\begin{abstract}
The space experiment CoRoT will provide continuous monitoring and high accuracy light curves of about sixty thousand stars. Selected binary systems will be observed in the Additional Program frame as targets of long and continuous pointed observations. Moreover, thousands of new binaries will certainly be detected and hundreds of them will have extremely accurate light curves. This will allow studies of fine effects on the light curves, monitoring of stellar activity and, in combination with ground-based observations, will provide exquisite determination of stellar parameters. Among the new discoveries of interesting systems of special value will be those of low mass binaries.
\end{abstract}

Keywords: CoRoT, close binaries, asteroseismology

\section{CoRoT in a nutshell}

CoRoT (COnvection, ROtation and planetary Transits) is a frenchled international "small" space mission whose launch is scheduled for August 2006. The mission ${ }^{1}$ focuses on two parallel "core programs", asteroseismology and extra-solar planet search, both requiring high accuracy photometry and continuous monitoring.

The CoRoT payload consists of a $28 \mathrm{~cm}$ telescope $(1200 \mathrm{~mm}$ focal length and field of view of 3.8 degrees) which will fly in a polar inertial orbit. Its focal plane hosts two pairs of $2 \mathrm{k} \times 2 \mathrm{k}$ CCDs, one dedicated to asteroseismology, the other to planetary transit searches, both have a field of view of $2.64^{\circ} \times 1.32^{\circ}$.

The defocused seismology field (PSF of 450 pixel for a G2 star) will observe a maximum of ten bright targets per run $(5.7 \leq \mathrm{V} \leq 9.5)$ with typical time sampling of $32^{\mathrm{S}}$. Simultaneously, the (focused) exo-planet field will observe up to 12000 fainter targets $(11.5 \leq \mathrm{V} \leq 16)$ with typical time sampling of $512^{\mathrm{s}}$. Color information will be available for the exoplanet targets brighter than $\mathrm{V}=15$, thanks to a prism providing very low resolution spectra. The expected accuracy of the photometry is a few parts per million (ppm) for asteroseismology and $100 \mathrm{ppm}$ for planet search.

\footnotetext{
${ }^{1}$ complete information on the mission is available at http://corot.oamp.fr
} 
The nominal mission duration is 2.5 years. Each year will be split in two $150^{\mathrm{d}}$-long "Long Runs" (LR), and two/one 20/30d_long "Short Runs" (SR). Pointing is restricted to the CoRoT "Eyes", i.e. two $10^{\circ}$ radius circles centered at $\delta=0$ and, respectively, $\alpha=6^{\mathrm{h}} 50^{\mathrm{m}}$ for the "Anticenter" direction and $\alpha=18^{\mathrm{h}} 50^{\mathrm{m}}$ for the opposite, "Center", direction. The LR fields (much smaller than the "Eyes") are chosen according to the best compromise between the two core programs, and are constrained to a short list of preselected seismology "primary targets". Short Runs can instead be anywhere inside the Eyes.

Science outside the two core programs is included in the mission as specific "Additional Programs" (APs), which can apply for both LR and SR observations. In practice, most APs will be performed in the Exo-planet field, because of the much larger number of available windows.

\section{CoRoT and close binaries}

The best CoRoT assets are the accurate and stable photometry and the continuous monitoring, with an estimated duty cycle of $94 \%$. These will provide close binary light curves of exquisite accuracy, excellent phase coverage and extending over a long time baseline.

The high accuracy with allow a thorough study of fine effects in the light curves by direct determination of second order effects such as limb darkening and gravity darkening. The latter can be derived for non eclipsing system as well, as it will clearly show up in the frequency domain.

The left panel of Fig.1 shows, as an example, the effect of different limb darkening laws on the light curve of a moderately close system. The synthetic light curves were computed by the 2003 version of the Wilson and Devinney code (Wilson and Devinney, 1971). For illustration purposes, we chose a model corresponding to the binary V805 Aql studied by Popper (1984); the main physical parameters of the model are: orbital period $\mathrm{P}=2.41^{\mathrm{d}}$, eccentricity $e=0$, inclination $i=86^{\circ}$, mass ratio $q=m_{s} / m_{p}=0.81$ (the index p stays for primary and refers to the more massive component), fractional component radii $r_{p}=0.18$ and $r_{s}=0.15$, effective temperatures $T_{p}=8184$ and $T_{s}=7178 \mathrm{~K}$, fractional V-band luminosity $L_{s} / L_{p}=0.362$. The figure shows the difference in magnitude between synthetic light curves obtained with different assumptions on limb darkening (different values of the coefficient for the linear law, or different laws). The difference between linear 

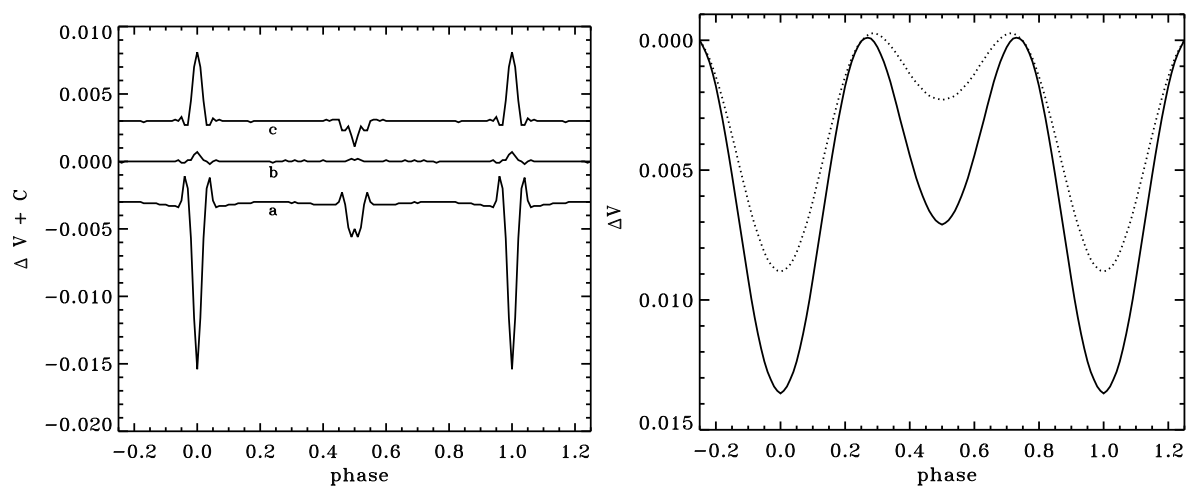

Figure 1. Left panel: difference between synthetic light curve models with different assumptions on the limb darkening law: a) linear $(\mathrm{x}=0.57)$ - linear $(\mathrm{x}=0.67), \mathrm{b})$ linear $(\mathrm{x}=0.57)$ - logarithmic, c) logarithmic law - square root law. The parameters of the non-linear laws are taken from Van Hamme (1993) for the corresponding component temperatures and gravities. For better readability curves a) and c) are shifted with respect to the zero line. Right panel: the effect of gravity darkening in a system with the same parameters as V805 Aql, but with $i=65^{\circ}$. The continuous line corresponds to a gravity darkening exponent $\beta=1.0$ (typical choice for radiative envelopes), the broken line to $\beta=0.32$ (convective envelopes).

laws of the form:

$$
R_{\lambda}(\mu)=\frac{I_{\lambda}(\mu)}{I_{\lambda}(1)}=1-x_{\lambda}(1-\mu)
$$

with $\mu=\cos \theta$, is obtained by changing the value of the coefficient $x_{\lambda}$. Among the proposed non-linear functional forms we considered a logarithmic relation:

$$
R_{\lambda}(\mu)=1-x_{\lambda}(1-\mu)-y_{\lambda} \mu \ln \mu
$$

and a square root one:

$$
R_{\lambda}(\mu)=1-x_{\lambda}(1-\mu)-y_{\lambda}(1-\sqrt{\mu})
$$

see Van Hamme (1993) and references therein. The coefficients used to compute the differences (b) and (c) in Fig. 1 have be taken, according to the component temperatures and gravities, from the tables by Van Hamme (1993) (i.e. the differences are between two fits of the same atmosphere). For this reason the deviations are smaller than in the case of linear laws. At any rate the photometry by CoRoT, with its $10^{-3}$ $10^{-4}$ mag accuracy, should allow to distinguish at least between linear and non-linear limb darkening prescriptions. The directly determined limb darkening can then be used to constrain model atmosphere details.

The right panel of Fig. 1 shows the amplitude of the ellipsoidal variation in a system differing from V805 Aql only for a lower value of the 

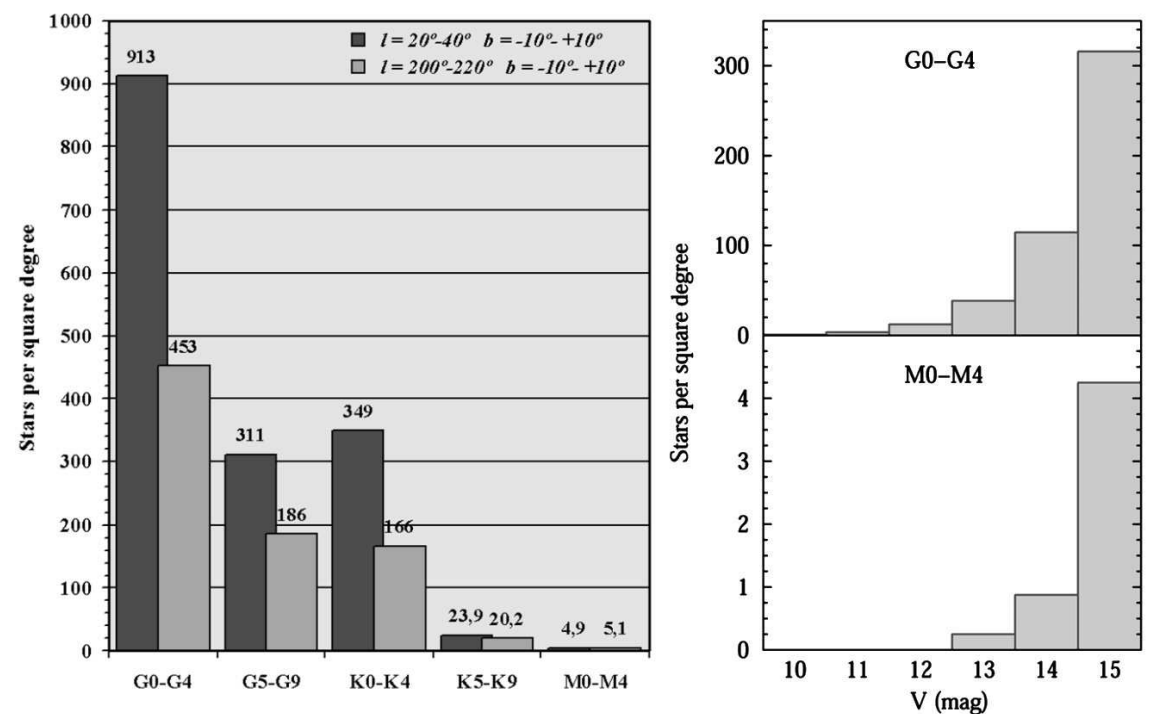

Figure 2. Left panel: the expected number of G-M stars of luminosity class IV-V, in a $16^{\square}$ field inside the CoRoT eyes. Right panel: the distribution in apparent magnitude in the winter (AC) field for G0-G4 and M0-M4 spectral type intervals.

inclination (to avoid eclipses), $i=65^{\circ}$. In spite of the small deviation from spherical symmetry (the maximum difference in radius for the primary component is $<2 \%$ ) the effect is large enough to be easily measured by CoRoT. The determination of gravity brightening and, with the help of follow-up observations, of the related system configuration, will yield precious information on close binary tidal evolution.

The long monitoring of the same field will allow to study on a long time baseline the manifestations of stellar activity such as spots, flares, stellar activity cycles. These will provide information on rotational period and differential rotation (from spot migration) in late-type components.

A very young field, asteroseismology in close binaries, combining the information from asteroseismology with that from eclipses will certainly profit of CoRoT assets. Looking for (solar-type) oscillations in suitable eclipsing binaries would have the advantage of knowing the masses, the radii and the orbit inclination angle, and therefore the rotational velocity. While binarity might increase the complexity of analysis, the identification of even non-radial pulsation modes is possible, as recently shown by several authors (Mkrtichian et al., 2005; Gamarova et al., 2005; Escolà-Sirisi et al., 2005). In particular eclipsing systems, with their near equator-on view, favor the detection of sectorial modes (i.e modes with $\ell=|m|$, where nodal lines form lines of longitude). 
Table I. Expected binaries per $3.4^{\square}$ field

\begin{tabular}{cc}
\hline Spectral type & expected EB number \\
\hline G0-G4 & $7.2-31.1$ \\
G5-G9 & $3.2-10.5$ \\
K0-K4 & $2.9-11.9$ \\
K5-K9 & $0.35-0.8$ \\
M0-M4 & $0.09-0.17$ \\
\hline
\end{tabular}

The CoRoT eyes contain $\sim 200$ known system, but only a tiny fraction of them will actually be observed during a Long Run ${ }^{2}$. However, many interesting systems will certainly be discovered in the CoRoT fields. Figure 2 shows the results of a simulation with the Besançon galaxy model (Robin et al., 2003), the constraints are: spectral type G-M, luminosity class IV-V, V magnitude 5.5-15.5 (to include both sismo- and exo-fields). The results give the number of star expected in a field of $4^{\circ} \times 4^{\circ}$ in the CoRoT Center and Anticenter directions. The magnitude distribution of the expected stars at the spectral extremes, and for the winter (Anticenter) field, is also shown.

Photometric large surveys such as OGLE, MACHO, ASAS, STARE, Vulcan indicate that $0.5 \%-1 \%$ of all monitored stars turn out to eclipsing binaries. From these percentages, and the density estimate, we obtained the maximum and minimum number of eclipsing binaries for an exo-planet field $\left(\sim 3.4^{\square}\right)$. Table I gives the results subdivided by spectral type intervals. With a total number of five LRs and five/ten SRs some hundreds of new objects are expected only along the MS. Among those, the stars at the two extremes in mass will be especially valuable to better constrain the theoretical models. In particular, at the low mass end of the MS, the absolute elements obtainable by combining follow-up spectroscopy with the CoRoT photometry will be precise enough to clarify what appears as a serious discrepancy in the mass-radius relation between theoretical models and data from observed eclipsing binaries (see Ribas talk, these proceedings).

In summary the "small space experiment" CoRoT will provide great opportunities not only in the field of asteroseismology and extra-solar planet searches. Research on binary stars (and more generally on stellar physics) will greatly benefit of its accurate photometry and stable mon-

\footnotetext{
${ }^{2}$ see the link 'Information' of the CoRoT Thematic Team on binaries webpage, at http://thor.ieec.uab.es/binteam/, for details about the known binaries in the "Eyes" and in the fields recently chosen for the first two Long Runs.
} 
itoring and many long standing questions will hopefully find a definite and clear answer.

\section{Acknowledgements}

We thank the members of the CoRoT "Thematic Team" on binaries for useful discussion on the different aspects of binary research that can be addressed by the mission. CM acknowledges the support from the MIUR-Cofin 2004 "Asteroseismology".

\section{References}

Escolà-Sirisi, E., Juan-Samsó, J., Vidal-Sáinz, J., Lampens, P., García-Melendo, E., Gómez-Forrellad, J. M., and P. Wils: 2005, 'Detection of a classical $\delta$ Scuti star in the new eclipsing binary system HIP 7666', Astronomy and Astrophysics 434, pp. 1063-1068

Gamarova, A. Yu., Mkrtichian, D. E. and E. Rodríguez: 2005, 'Mode identification in the ecplising binary systems with primary pulsating components', ASP Conf. Ser. 333: Tidal Evolution and Oscillations in Binary Stars 333, pp. 258

Mkrtichian, D. E., Rodríguez, E., Olson, E. C., Kusakin, A. V., Kim, S.-L., Lehmann, H., Gamarova, A. Yu., and Y. W. Kang : 2005, 'Pulsations in eclipsing binaries', ASP Conf. Ser. 333: Tidal Evolution and Oscillations in Binary Stars 333, pp. 197

Popper, D. M.: 1984, 'Error analysis of light curves of detached eclipsing binary systems', Astronomical Journal, 89, pp. 132-144

Robin, A. C., Reylé, C., Derrière, S., and S. Picaud: 2003, 'A synthetic view on structure and evolution of the Milky Way', Astronomy and Astrophysics 409, pp. $523-540$

Van Hamme, W.: 1993, 'New limb-darkening coefficients for modeling binary star light curves', Astronomical Journal 106, pp. 2096-2117

Wilson, R. E. and E.J. Devinney: 1971, 'Realization of Accurate Close-Binary Light Curves: Application to MR Cygni', Astrophysical Journal 166, pp. 605 